\title{
Patient-specific outcome simulation after surgical correction of Pectus Excavatum: a preliminary study
}

\author{
Mafalda Couto $^{\mathrm{a}, \mathrm{b}, \# \text {, João Gomes-Fonseca }}{ }^{\mathrm{a}, \mathrm{b}, \#, *}$, António H. J. Moreira ${ }^{\mathrm{c}}$, Tiago Henriques-Coelho ${ }^{\mathrm{d}, \mathrm{e}, \mathrm{f}}$, \\ Jaime C. Fonseca ${ }^{\mathrm{g}, \mathrm{h}}$, António C.M. Pinho ${ }^{\mathrm{i}}$, Jorge Correia-Pinto ${ }^{\mathrm{a}, \mathrm{b}, \mathrm{j}}$, João L. Vilaça ${ }^{\mathrm{c}}$
}

${ }^{a}$ Life and Health Sciences Research Institute (ICVS), School of Medicine, University of Minho, Braga, Portugal; ' ICVS/3B's - PT Government Associate Laboratory, Braga/Guimarães, Portugal; '2Ai, Polytechnic Institute of Cávado and Ave, Barcelos, Portugal; ${ }^{\mathrm{d} D e p a r t m e n t ~ o f ~ P e d i a t r i c ~ S u r g e r y, ~}$ Centro Hospitalar de São João, Porto, Portugal; ${ }^{\mathrm{C} D e p a r t m e n t ~ o f ~ P e d i a t r i c s, ~ F a c u l t y ~ o f ~ M e d i c i n e, ~}$ University of Porto, Porto, Portugal; ${ }^{\mathrm{f}}$ Cardiovascular R\&D Centre (UnIC), Faculty of Medicine, University of Porto, Porto, Portugal; ${ }^{\mathrm{g}}$ Algoritmi Center, School of Engineering, University of Minho, Guimarães, Portugal, ${ }^{\mathrm{h}}$ Department of Industrial Electronics, School of Engineering, University of Minho, Guimarães, Portugal; ${ }^{i}$ Department of Mechanical Engineering, School of Engineering, University of Minho, Guimarães, Portugal; ${ }^{j}$ Department of Pediatric Surgery, Hospital de Braga, Braga, Portugal;

\#These authors contributed equally to this work.

*Corresponding author: joaofonseca@med.uminho.pt

\begin{abstract}
Although minimally invasive Nuss procedure is frequently performed to correct Pectus Excavatum, the successful aesthetical outcome is not always ensured. Using the computed tomography (CT) data of six patients, high-quality surfaces of the anterior chest wall were generated, alongside with a personalized corrective-bar. Through finite element method (FEM), replicating the surgical procedure, a simulation of the anterior chest wall correction was conducted. The assessment of this methodology was verified by comparing the metrics from the real meshes (3D scanned before and after surgery) and simulated meshes (obtained before and after FEM). Results show a mean difference of $2.85 \pm 5.77 \mathrm{~mm}$ on the point of maximum correction between simulated and real outcomes. No statistical differences were found $(p=$ 0.281). High aesthetical similarity was observed concerning simulated and real outcomes. The proposed methodology presents a patient-specific simulation that may be used to plan, predict and improve the surgical outcome of the Nuss procedure. Further studies should continue to improve the presented methodology.
\end{abstract}

Keywords: aesthetic outcome prediction, finite element method, pectus excavatum, surgical simulation.

\section{INTRODUCTION}

Pectus Excavatum (PE) is a common chest wall deformity, with an incidence of 1 in 400 to 1 in 1000 births and a maleto-female prevalence of 3-5:1 ${ }^{1,2}$. This deformation is commonly characterized by the posterior depression of the sternum and adjacent thoracic structures ${ }^{3}$. Frequently observed in infancy and slowly progressing with growth, PE is stated as congenital in $90 \%$ of cases ${ }^{1,2}$. The deformity in the anterior chest wall may vary in depth, width, symmetry and sternum rotation, classified accordingly to the Haller index or the correction index for its severity ${ }^{4}$. Some severe cases reported cardiopulmonary difficulties or loss of appetite and weight, caused by the compression in the chest and stomach. However, the psychological health and self-worth caused by the aesthetical appearance is the leading reason to perform the PE correction ${ }^{5-7}$.

Currently, the Nuss surgical technique is a well-established procedure for the PE correction ${ }^{8-10}$. This technique involves the insertion of a prebent metal bar through two axillary incisions in the retrosternal space, elevating and remodeling the 
thoracic cage structures. The metal bar profile is defined according to the anatomical structures acquired from computed tomography (CT), including the degree and shape of the deformity of PE. The bar is removed after 2-4 years ${ }^{6,11}$.

Personalized simulation of surgical procedures has indisputable advantages in several fields of medicine and PE is no exception ${ }^{12-14}$. Along the years, several studies explored the Nuss procedure using FEM to analyze the chest wall evolution where three-dimensional (3D) models were generated from the reconstruction of CT data. Briefly, these studies focused on the: i) comparison of stress distribution between adult and child patients ${ }^{15}$; ii) analysis of the forces on the thoracic cage of asymmetric PE patients ${ }^{16}$; iii) dynamic effects on the spine of patients with asymmetric chest ${ }^{17}$; iv) correction bar positioning simulation ${ }^{18,19}$; v) effect of additional bar application on the patient's postoperative pain ${ }^{20}$; vi) generation of parametric models to create a simplification of a $3 \mathrm{D}$ chest model using $\mathrm{CT}$ measurements and chest surface scans ${ }^{21}$; vii) simultaneous correction of PE and scoliosis ${ }^{22}$; and, viii) methodologies to individually design and optimize the shape of the Nuss bar, advising the location of the incisions using CT data of the patient ${ }^{23}$. Although the relevant advances of these studies, we considered that different drawbacks are presented in each one, namely: the limited sample size (most studies are limited to 1-2 cases); the neglection of the inherent variability, properties, and specificity of each anatomical structure during simulation; the disregard of the skin as a crucial structure to verify the aesthetical outcome; the neglection of the real shape of the bar; and, the fact that the real surgical outcome was frequently not considered as the ground truth.

Therefore, this work proposes the introduction of an accurate patient-specific numerical simulation workflow to predict and improve the functional and visual outcome of the Nuss technique. In that sense, a methodology to numerically replicate the surgical procedure is carried out, using the data from six PE patients who underwent minimally invasive Nuss procedure. Real data acquired using 3D surface scanning is used to assess the simulated outcomes.

\section{MATERIALS AND METHODS}

The presented methodology was divided into three main steps: i) generation of the patient-specific biomechanical model; ii) FEM of the Nuss surgical procedure; and iii) comparison of simulated and real outcomes (see Figure 1).

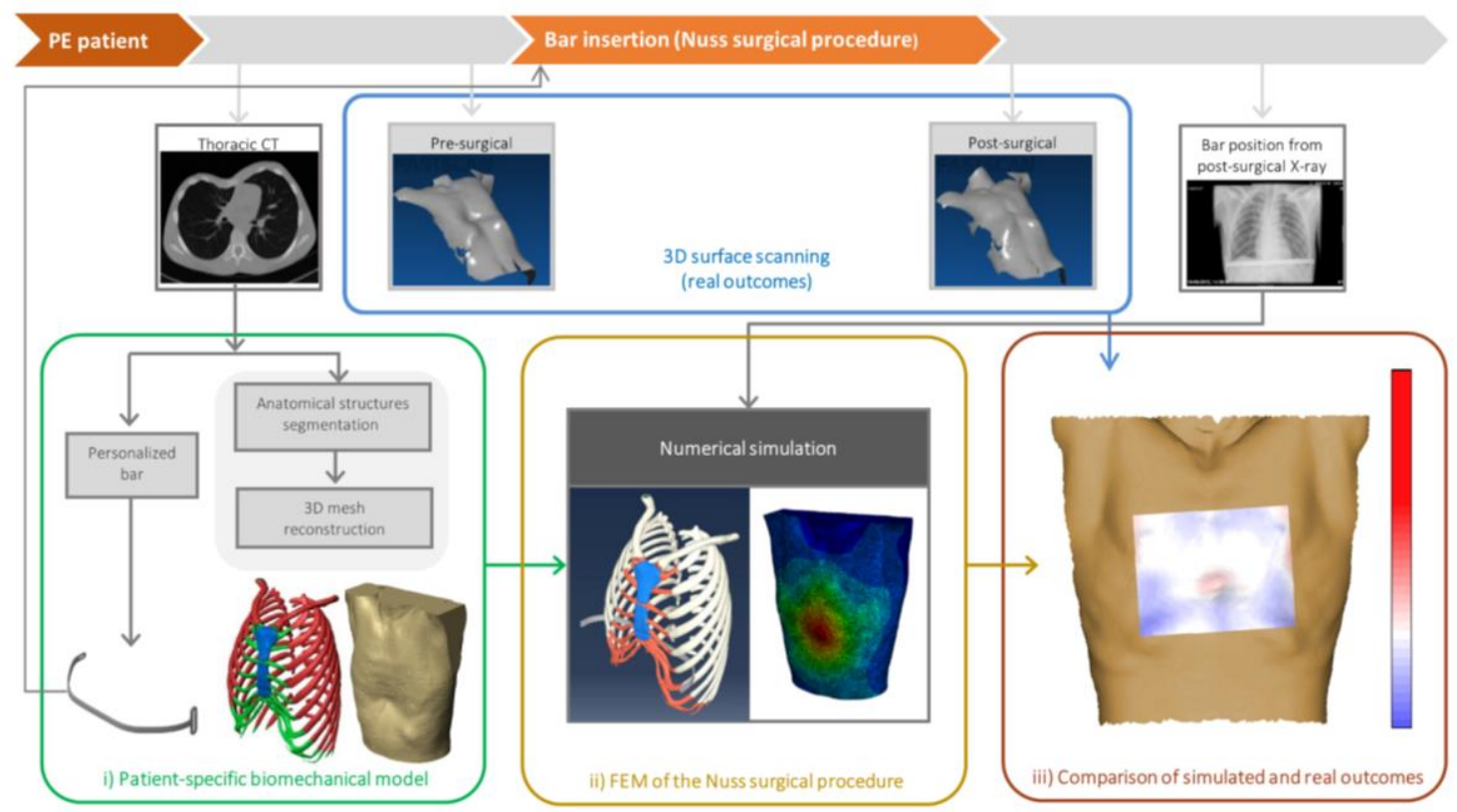

Figure 1 - Overview of the different stages of the proposed methodology to simulate the Nuss surgical procedure. Computed tomography (CT), X-Ray, and 3D surface scanning data were acquired before and after surgery. While the CT data was used to create the patient-specific biomechanical model of patient's chest structures and a personalized bar (same shape in simulation and surgery) (i), the X-ray data was used to detect the real position of the bar after the correction. Both are then inputted into the FEM of the Nuss surgical procedure (ii). Finally, the 3D surface scanning data was used to compare the outcomes of the FEM (iii). 


\subsection{Generation of the patient-specific biomechanical model}

The generation of the anatomical models of the patient's chest was divided into two main steps. First, the manual segmentation of the anatomical structures was performed using the CT data of each patient. Second, a 3D reconstruction of the chest was executed, and the mesh generation of the main thorax structures was accomplished.

For simulation purposes, the segmented anatomical structures consisted of ribs (divided into cortical and trabecular bone), sternum and costal cartilages, disregarding internal soft tissues, such as intercostal muscles and organs. According to previous works ${ }^{16,24}$, these segmented structures were used due to their major contribution to the thoracic cage integrity. Furthermore, based on clinical observations, the spinal structure does not significantly change immediately after the Nuss procedure, and was therefore not included in the FEM. Despite having a smaller contribution to the simulation result ${ }^{16}$, the skin was considered due to the main role in the final appearance of the anterior chest wall surface.

Regarding the material properties of both ribs (cortical/trabecular) and sternum, there has been a consensus in the field that bone structures are considered isotropic, homogeneous and linear elastic materials ${ }^{16,25}$. On the other hand, there is no agreement concerning the material properties of PE cartilages ${ }^{21}$, where studies suggested that age and calcification can influence the biomechanical properties ${ }^{26,27}$, which have the potential to affect its structural behavior ${ }^{28}$. In this sense, material properties for the bone and cartilage were considered isotropic, homogeneous and linearly elastic. Table 1 presents the elastic modulus $(\boldsymbol{E})$ and the Poisson ratio $(v)$ of the materials properties considered in the simulation.

The skin structure was defined as a single layer (including skin, muscles and other soft tissues), which contacts with the bone and cartilage structures. This structure assumes a passive role on FEM, where its motion is a consequence of the simulation outcome of other structures after the bar insertion. As proposed by Lapeer et al. ${ }^{29}$, here we also defined the skin as a hyper elastic material, where a dynamic mesh was set to fit the skin stress-strain.

The segmentation of the different structures from the CT images was performed manually using the 3D Slicer software (version 4.6) ${ }^{30}$. Then, the chest model was generated using iso2mesh version 2.0 developed by Fang et al. ${ }^{31}$. This toolbox enables the generation of 3D high-quality surface and volumetric mesh in MATLAB® (Mathworks Inc., Natick, Massachusetts).

The personalized bar was modeled with the system developed by Vilaça et al. ${ }^{32}$ using the CT data of each patient. The bar shape and size were used to bend the metal bar applied in the surgery and to create the virtual model of the bar used in the FEM simulation. The virtual bar was created in a computer-aided design software, i.e. Solidworks, (SolidWorks Corp., Concord, MA, USA) ${ }^{33}$. The material properties of titanium alloy Ti-6Al-4V were considered for the bar ${ }^{34}$.

\subsection{FEM of the Nuss surgical procedure}

FEM is a well-established technique in the field of biomechanics ${ }^{17}$. This method enables the assessment of global or local strains and displacements, simulating the practical problem and providing useful information from the clinical point-of-view ${ }^{16,18,35}$.

The patient-specific chest model and the personalized bar were imported into the FEM software, i.e. ABAQUS/Explicit (Dassault Systèmes, Vélizy-Villacoublay, France). For the interaction between the bar and the chest model, two different coefficients of friction were used: 0.65 for titanium with cortical bone ${ }^{36}$; and 0.15 for titanium with costal cartilage ${ }^{18}$.

Table 1 - Material properties used in the simulation

\begin{tabular}{|c|c|c|c|c|}
\hline & Cortical bone & Trabecular bone & Costal cartilage & Ti-6Al-4V bar \\
\hline $\boldsymbol{E}(\mathrm{MPa})$ & 11500.0 & 40.0 & 24.5 & 113764.0 \\
\hline$v$ & 0.300 & 0.450 & 0.400 & 0.342 \\
\hline Reference & Li et $a l .{ }^{23}$ & Li et al. ${ }^{23}$ & Awrejcewicz et al. ${ }^{22}$ & Rack et al. ${ }^{31}$ \\
\hline
\end{tabular}


Besides, the displacement of the joints between the rib and spine was assumed to be constant ${ }^{16}$, thus the ribs backend nodes were constrained as pinned (i.e. fixed translations).

Specifically, the Nuss procedure consists in the insertion of a prebent metal bar through a transverse incision made at both sides of the midaxillary line in the retro-sternum space. The bar with expanding surgical forceps is pulled in and dragged across the sternum rear with the convexity facing towards the back. Then, when the bar reaches the location, it is turned over, starting to generate the reshaping of the sternum and adjacent structures.

To reproduce the surgical procedure, the method was divided into two steps. First - bar set - based on the post-surgical patient X-ray information, the axial location of the bar was determined and displayed with the concave side of the bar posteriorly to the sternum (Figure 2 - a)). Then, the bar is rotated $180^{\circ}$ and pulled forward to promote the sternum and underlying structures displacement (Figure 2 - b) and c)). Second - release constrictions - once the bar is placed, forcing the structures to a new forward position, the displacement constraints are inactivated. At this point, due to the reaction forces and displacements generated from the intercostal introduction of the prosthesis, both bar and chest models adapt to the new position, achieving the post-surgical outcome. The output of this step is a pre-surgical (PreSIM) and a postsurgical (PostSIM) simulated meshes.

\subsection{Comparison of simulated and real surgical outcome}

Using a 3D handheld laser scanner, the Polhemus FastSCANTM Cobra (Polhemus, Colchester, VT), a cross-sectional depth profile of the patient's chest wall surface was acquired before (PreREAL) and after (PostREAL) the surgical procedure ${ }^{37}$. Following the methodology presented by Gomes-Fonseca et al., the acquired data was processed to improve the quality of the chest meshes, preserving the surface details with high accuracy ${ }^{38}$.

The assessment was performed comparing the 3D surfaces obtained from the FEM and 3D surface scanning. Thus, based on Gomes-Fonseca et al. as well, a registration technique was conducted (based on the iterative closest point method) to overlap the surfaces from different moments, and then the signed distance between surfaces was computed. The displacement analysis was performed and restricted to an area from the inter-mammary (between nipples) and mammillary lines to the end of the sternum (see Figure 1 and Figure 3), measuring the distance differences resulting from the bar insertion. Therefore, PreSIM vs. PostSIM (i.e. pre and post-simulation differences), PreREAL vs. PostREAL (i.e. pre and post-surgical differences), and PostSIM vs. PostREAL (post-simulation and post-surgical differences) were computed, and the maximum and mean distances were obtained.

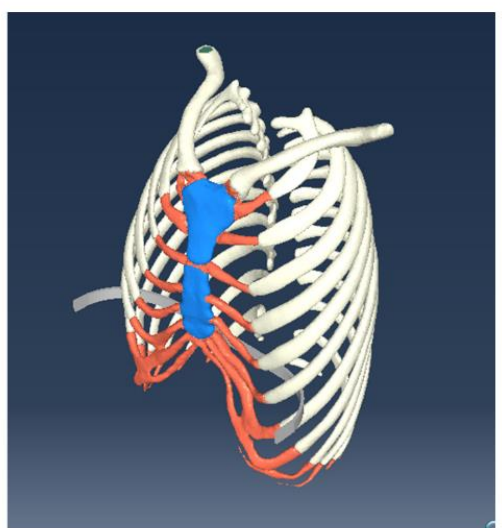

a)

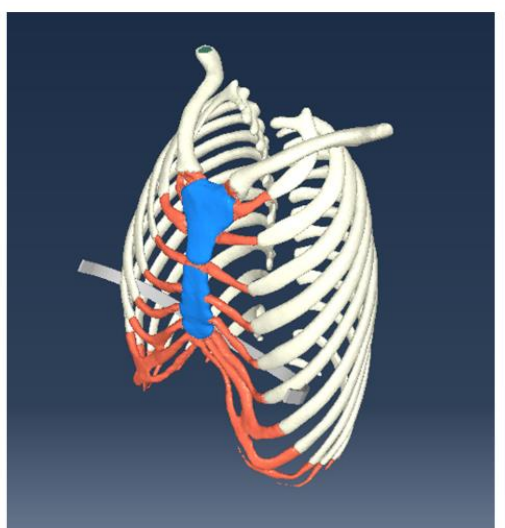

b)

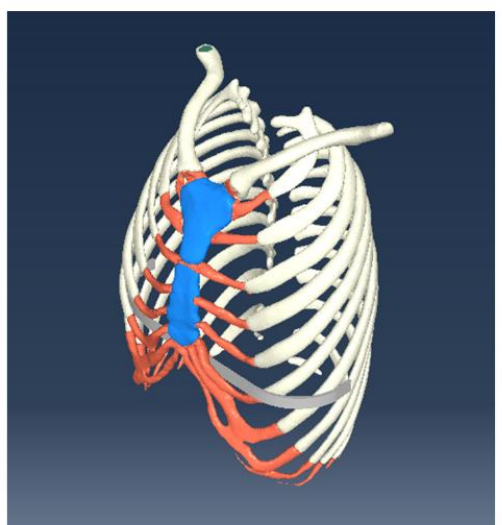

c)

Figure 2 - Representation of bar rotation applied in minimally invasive Nuss procedure and simulated using FEM. a) Bar positioned at real axial location before rotation. b) Time point during $180^{\circ}$ bar rotation. c) Bar position after rotation showing chest reshaping. 


\subsection{Study sample}

Six male patients with symmetric or very light asymmetric deformation were considered for the methodology assessment. The mean age of these patients was $15 \pm 1$ years (range: 14 to 16) with a Haller Index of $3.29 \pm 0.89$ (range: 2.45 to 4.97). CT image size was $512 \times 512$ pixels with a slice thickness of $3 \mathrm{~mm}$. The pixel resolution ranged from 0.50 to $0.73 \mathrm{~mm}$ and 0.46 to $0.84 \mathrm{~mm}$, for both vertical and horizontal directions, respectively.

\subsection{Statistical analysis}

All statistical tests were conducted using MATLAB ${ }^{\circledR}$ (version R2016b, The Mathworks Inc., Natick, MA). The assumption of normality was assessed for all variables and, based on the results, parametric or nonparametric tests were applied accordingly. The effect size and statistical significance were reported. Correlation and regression analysis were

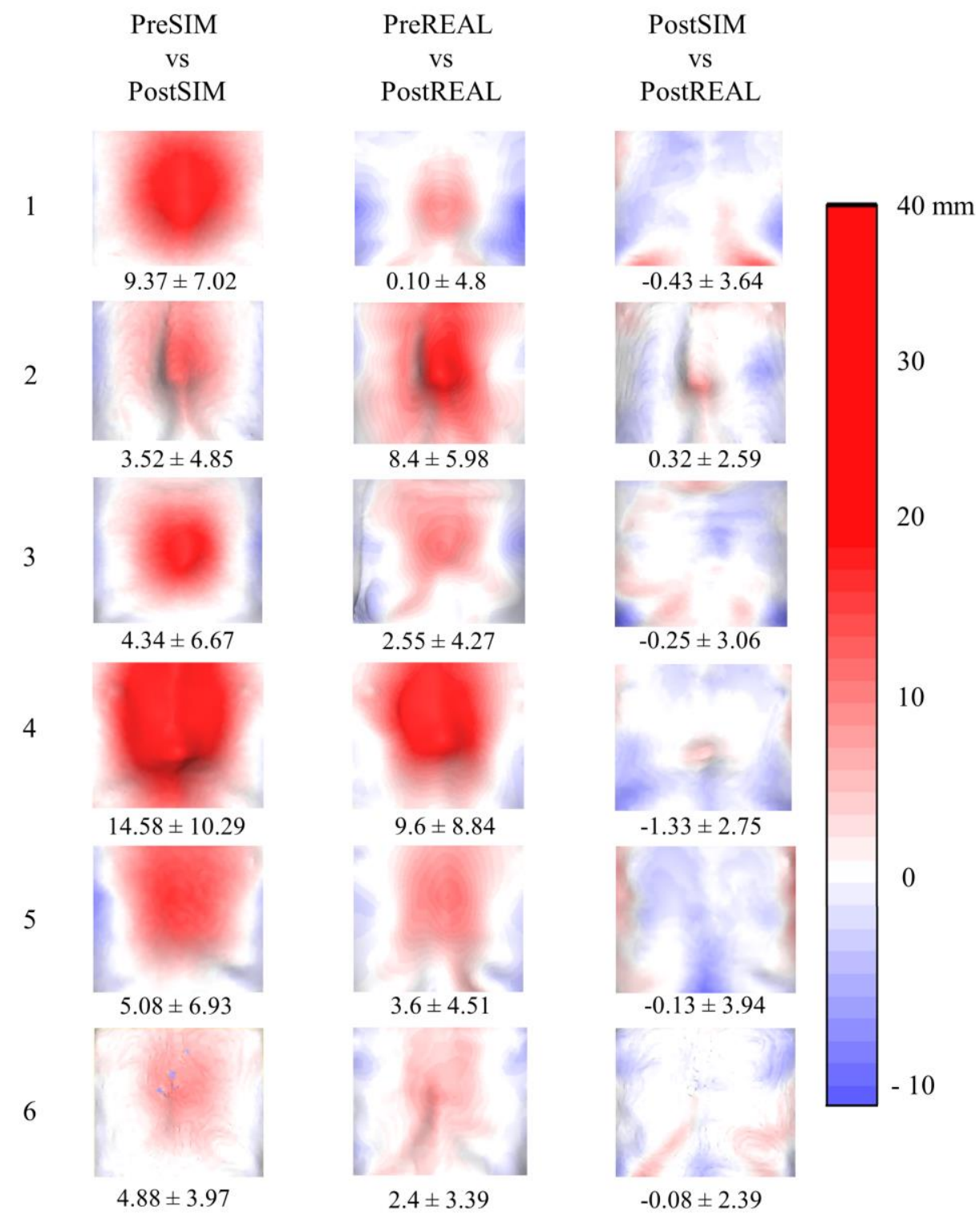

Figure 3 - Representation of mean distances between PreSIM mesh vs. PostSIM mesh; PreREAL mesh vs. PostREAL mesh; and PostSIM mesh vs. PostREAL mesh. Mean and standard deviation are reported for the six cases and for all comparisons. Red means chest elevation, while blue means chest depression. 
also performed. All statistics were considered significant if $p<0.05$.

\section{RESULTS}

The results are summarized in Figure 3. Shape differences between pre- and post-surgical meshes were clearly visible in both simulated (i.e. PreSIM vs. PostSIM) and real ones (i.e. PreREAL vs. PostREAL), where the elevation of the internal structures (i.e. sternum and costal cartilages) were present after bar insertion. While the average distances of PreSIM $v s$. PostSIM and PreREAL vs. PostREAL presented differences, PostSIM vs. PostREAL were similar. Indeed, mean distances were closed to zero in most cases.

The average value of the maximum distance obtained in PreSIM vs. PostSIM and PreREAL vs. PostREAL was $21.44 \pm$ $9.39 \mathrm{~mm}$ and $18.59 \pm 6.22 \mathrm{~mm}$, respectively, with a mean difference of $2.85 \pm 5.77 \mathrm{~mm}$ (see Table 2). No statistical differences between measurements were found when a paired sample $t$-test was performed $(p=0.281)$, although a medium effect size was obtained (Cohen's $d=0.490$ ).

A strong and positive Pearson's correlation was found $(\mathrm{r}(6)=0.801)$, being almost significant $(p=0.055)$. The regression analysis revealed that the maximum elevation point of the chest was approximately $21 \%$ higher in simulated meshes than in real ones (Figure 4).

\section{DISCUSSION}

In this work, we presented a new methodology based on a patient-specific simulation that may be used to plan, predict and improve the surgical outcome of the Nuss procedure. To the best of our knowledge, this was the first study that performed a numerical simulation of the Nuss surgical procedure using a personalized bar, with the same shape as the one that was inserted during the surgery. Beyond that, this study also validated the simulation outcomes using real data from the patients.

We collected data from six PE patients who underwent minimally invasive Nuss procedure. For all cases the same

Table 2 - Maximum distance computed between simulated (PreSIM vs PostSIM) and real outcomes (PreREAL vs PostREAL) for the six PE cases.

\begin{tabular}{|l|l|l|l|l|l|}
\hline \multicolumn{2}{|c|}{} & \multicolumn{3}{c|}{ Maximum distance (mm) } \\
\hline Case & Age (Y) & $\begin{array}{c}\text { Haler } \\
\text { Index }\end{array}$ & $\begin{array}{c}\Delta \text { (PreSIM } \text { vs. } \\
\text { PostSIM) }\end{array}$ & $\begin{array}{c}\Delta \text { (PreREAL } \text { vs. } \\
\text { PostREAL) }\end{array}$ & Differences \\
\hline $\mathbf{1}$ & 15 & 2.75 & 23.91 & 16.62 & 7.29 \\
\hline $\mathbf{2}$ & 14 & 3.90 & 13.16 & 20.64 & -7.48 \\
\hline $\mathbf{3}$ & 16 & 2.45 & 23.62 & 17.74 & 5.88 \\
\hline $\mathbf{4}$ & 14 & 4.97 & 37.58 & 29.55 & 8.03 \\
\hline $\mathbf{5}$ & 15 & 3.10 & 18.55 & 16.04 & 2.51 \\
\hline $\mathbf{6}$ & 16 & 2.55 & 11.80 & 10.96 & 0.84 \\
\hline Mean \pm SD & $15 \pm 0.82$ & $3.29 \pm 0.89$ & $21.44 \pm 9.39$ & $18.59 \pm 6.22$ & $2.85 \pm 5.77$ \\
\hline \multicolumn{2}{|l|}{ Paired sample t-test } & $\mathrm{t}(5)=1.207, p=0.281$, Cohen's $d=0.490$ & \\
\hline
\end{tabular}




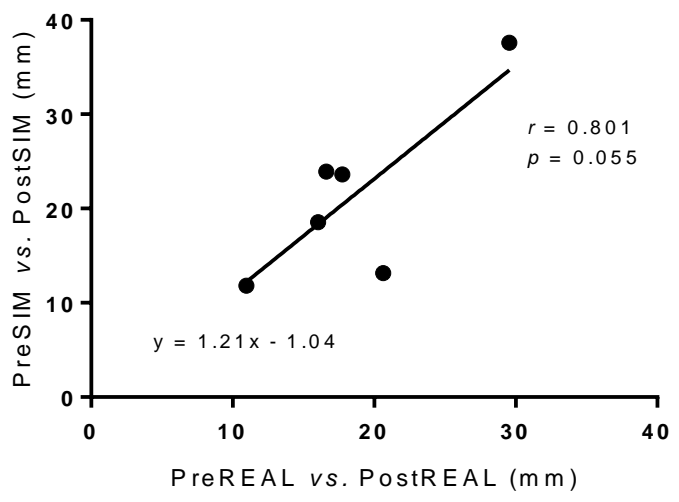

Figure 4 - Correlation and regression analysis using maximum distance between simulated (i.e. PreSIM vs. PostSIM) and real (PreREAL vs. PostREAL) outcomes.

gender was considered, low age variability and low asymmetry, being the degree of depression the main variable.

3D shape differences before and after surgery were observed in both simulated and real surfaces outcomes, which means that the displacement induced by the bar insertion was observed in both. Magnitude differences between simulated and real outcomes were present, where the simulation showed superior values in most cases. Despite no statistical differences were found, the effect size and, particularly, the regression analysis revealed that simulations were approximately $21 \%$ higher than real outcomes. However, when the post-surgical meshes (PostSIM vs. PostREAL) were aligned and compared, the mean differences were approximately zero (Figure 3 ). These results indicate that the displacement values present differences, but the chest shape was similar after the numerical simulation and surgery, as it is visible in Figure 5 . From the visualization perspective, it can be observed that small defects and features of the real correction were present in the simulation outcome. Furthermore, the points where the maximum displacement was observed were approximately coincident in all comparisons.

A limitation of this assessment was to establish the best mesh alignment. An evident 3D shape difference is observed between pre-surgical and post-surgical meshes, which may hamper the exact alignment, and consequently the computed values. Besides, differences between meshes can be caused by the position and orientation changes between acquisition moments, due to chest wall height and width increase during bar insertion, and respiratory movements during data acquisition. Thus, the used methodology tried to minimize the errors of these alignments. However, it must be mentioned that other alignment techniques could achieve different results.

The goal of this work was to submit non-parametric 3D models to the real effects of the bar insertion. However, to accomplish the simulation, since the application of FEM is computationally demanding, this work proposed a set of simplifications which may entail an error to the analyzed outcome. Even though, the proposed strategy for FEM, where different materials properties for the bone, costal cartilages, and skin were considered leading to independent structures during simulation, brought benefits to the final outcomes.

\section{CONCLUSIONS}

A patient-specific simulation that might be used to plan, predict and improve the surgical outcome of the Nuss procedure was presented and preliminarily assessed. The morphological similarity observed in the post-surgical meshes indicated the reliability of the aesthetical outcome prediction of the presented methodology. Thus, being the PE correction different for each patient, the chest deformity appearance had specific characteristics that were differently simulated in the outcome. Therefore, the proposed methodology allowed the pre-visualization of the outcome which may distinctively improve the patient confidence in the Nuss surgical procedure. Further studies should continue to improve the presented methodology. 


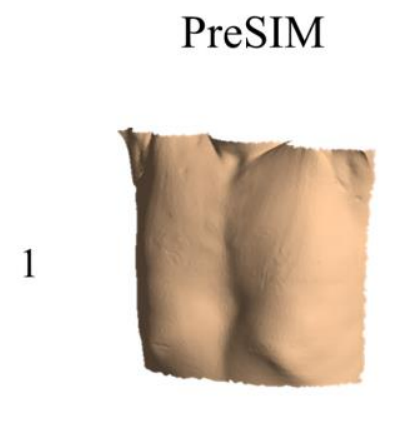

$$
\text { PostSIM PostREAL }
$$
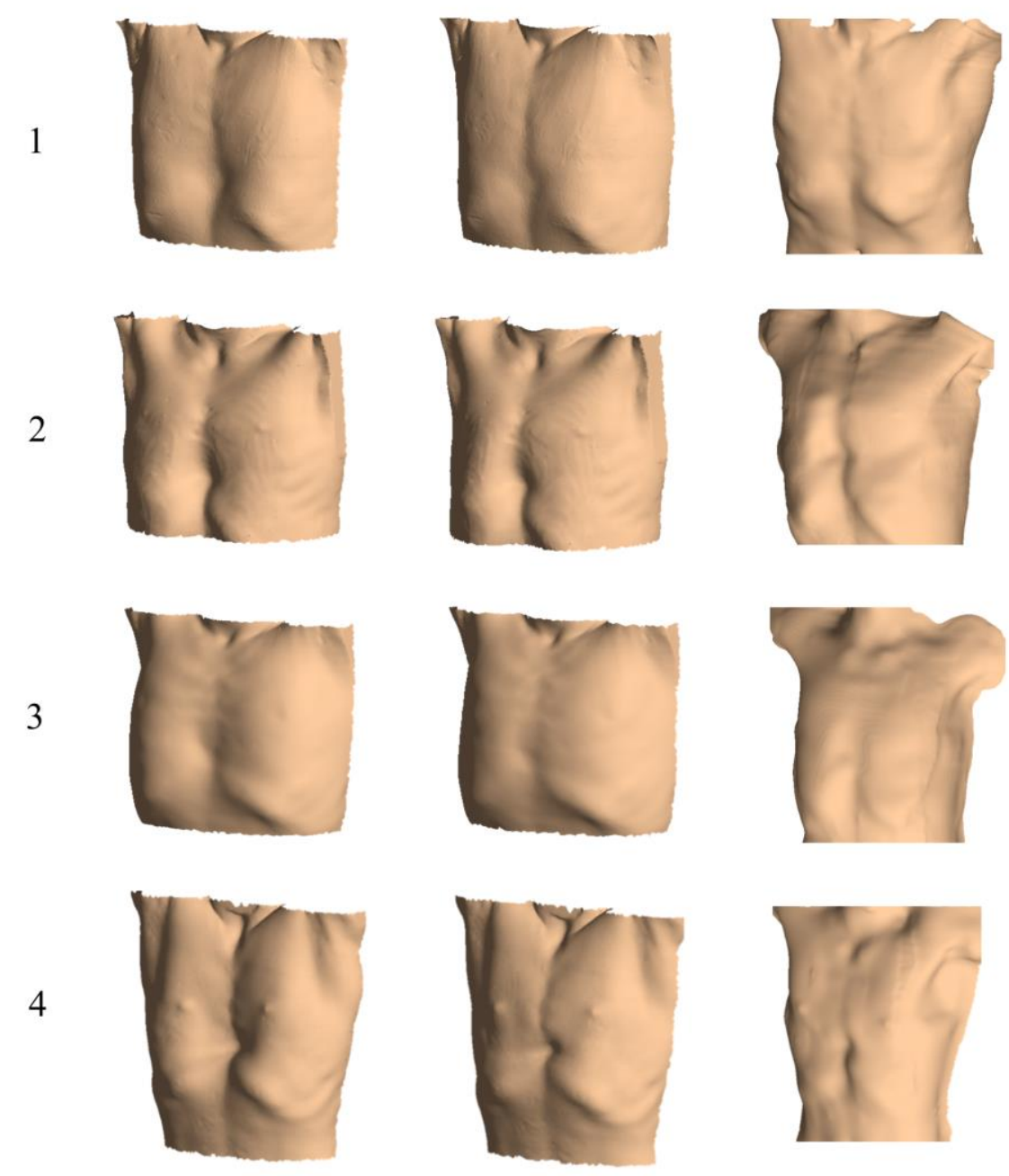

5
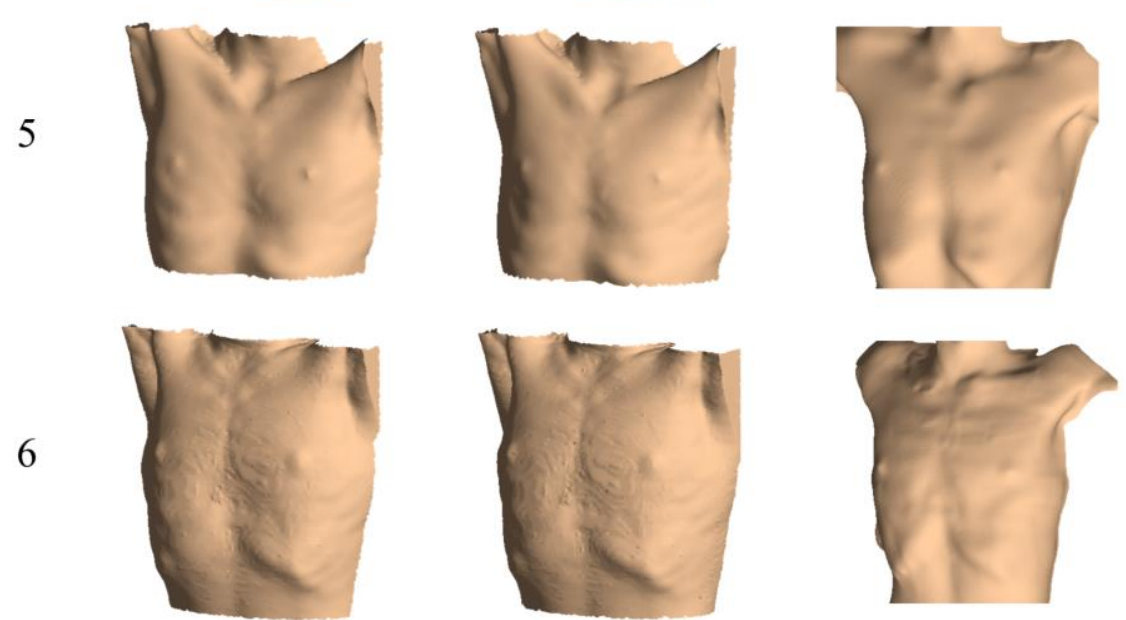

Figure 5 - Isometric views of PreSIM, PostSIM, PostREAL meshes for all patients. 


\section{ACKNOWLEDGMENTS}

This work has been funded by FEDER funds, through the Competitiveness Factors Operational Programme (COMPETE), and by National funds, through the Foundation for Science and Technology (FCT), under the scope of the projects POCI-01-0145-FEDER-007038; NORTE-01-0145-FEDER-000013; and NORTE-01-0145-FEDER-024300, supported by the Northern Portugal Regional Operational Programme (NORTE 2020), under the Portugal 2020 Partnership Agreement, through the European Regional Development Fund (FEDER). João Gomes-Fonseca was funded by FCT under the Ph.D. grant PD/BDE/113597/2015.

The funders had no role in study design, data collection, and analysis, decision to publish, or preparation of the manuscript. The authors would like to thank the departments of pediatric surgery of Centro Hospitalar São João and Hospital of Braga.

\section{REFERENCES}

[1] Goretsky, M. J., Kelly, R. E., Croitoru, D. and Nuss, D., "Chest wall anomalies: pectus excavatum and pectus carinatum.," Adolesc. Med. Clin. 15, 455-471 (2004).

[2] Aloi, I., Braguglia, A. and Inserra, A., "Pectus excavatum," Paediatr. Child Health (Oxford). 19, S132-S142 (2009).

[3] Peña, A., Pérez, L., Nurko, S. and Dorenbaum, D., "Pectus carinatum and pectus excavatum: are they the same disease?," Am. Surg. 47, 215-218 (1981).

[4] St. Peter, S. D., Juang, D., Garey, C. L., Laituri, C. A., Ostlie, D. J., Sharp, R. J. and Snyder, C. L., “A novel measure for pectus excavatum: The correction index," J. Pediatr. Surg. 46(12), 2270-2273 (2011).

[5] Einsiedel, E. and Clausner, A., "Funnel chest. Psychological and psychosomatic aspects in children, youngsters, and young adults.," J. Cardiovasc. Surg. (Torino). 40, 733-736 (1999).

[6] Iida, H., "Surgical repair of pectus excavatum.," Gen. Thorac. Cardiovasc. Surg. 58, 55-61 (2010).

[7] Roberts, J., Hayashi, A., Anderson, J. O., Martin, J. M. and Maxwell, L. L., "Quality of life of patients who have undergone the Nuss procedure for pectus excavatum: Preliminary findings.," J. Pediatr. Surg. 38, 779-783 (2003).

[8] Nuss, D., Croitoru, D. P., Kelly, R. E., Goretsky, M. J., Nuss, K. J. and Gustin, T. S., "Review and discussion of the complications of minimally invasive pectus excavatum repair.," Eur. J. Pediatr. Surg. 12, 230-234 (2002).

[9] Nuss, D., "Minimally invasive surgical repair of pectus excavatum," Semin. Pediatr. Surg. 17(3), 209-217 (2008).

[10] Notrica, D. M., McMahon, L. E., Johnson, K. N., Velez, D. A., McGill, L. C. and Jaroszewski, D. E., “LifeThreatening Hemorrhage During Removal of a Nuss Bar Associated With Sternal Erosion,” Ann. Thorac. Surg. 98(3), 1104-1106 (2014).

[11] Hosie, S., Sitkiewicz, T., Petersen, C., Göbel, P., Schaarschmidt, K., Till, H., Noatnick, M., Winiker, H., Hagl, C., Schmedding, A. and Waag, K.-L., "Minimally invasive repair of pectus excavatum--the Nuss procedure. A European multicentre experience.," Eur. J. Pediatr. Surg. 12, 235-238 (2002).

[12] Handels, H., Ehrhardt, J., Plötz, W. and Pöppl, S. J., "Three-dimensional planning and simulation of hip operations and computer-assisted construction of endoprostheses in bone tumor surgery.," Comput. Aided Surg. 6, 65-76 (2001).

[13] Vilaça, J. L., Moreira, A. H. J., L-Rodrigues, P., Rodrigues, N., Fonseca, J. C., Pinho, A. C. M. and CorreiaPinto, J., "Virtual simulation of the postsurgical cosmetic outcome in patients with Pectus Excavatum," Proc. SPIE 7964, Med. Imaging 2011 Vis. Image-Guided Proced. Model., K. H. Wong and D. R. Holmes III, Eds., 79642L-79642L-15, Lake Buena Vista, Florida (2011).

[14] Moreira, A. H. J., Rodrigues, P. L., Fonseca, J., Pinho, A. C. M., Rodrigues, N. F., Correia-Pinto, J. and Vilaça, J. L., "Pectus excavatum postsurgical outcome based on preoperative soft body dynamics simulation," Proc. SPIE 8316, Med. Imaging 2012 Image-Guided Proced. Robot. Interv. Model., D. R. Holmes III and K. H. Wong, Eds., 83160K-83160K-8, San Diego, California, USA (2012).

[15] Nagasao, T., Miyamoto, J., Tamaki, T., Ichihara, K., Jiang, H., Taguchi, T., Yozu, R. and Nakajima, T., "Stress distribution on the thorax after the Nuss procedure for pectus excavatum results in different patterns between adult and child patients.," J. Thorac. Cardiovasc. Surg. 134, 1502-1507 (2007). 
[16] Chang, P. Y., Hsu, Z.-Y., Chen, D.-P., Lai, J.-Y. and Wang, C.-J., "Preliminary analysis of the forces on the thoracic cage of patients with pectus excavatum after the Nuss procedure.," Clin. Biomech. (Bristol, Avon) 23, 881-885 (2008).

[17] Nagasao, T., Noguchi, M., Miyamoto, J., Jiang, H., Ding, W., Shimizu, Y. and Kishi, K., "Dynamic effects of the Nuss procedure on the spine in asymmetric pectus excavatum.," J. Thorac. Cardiovasc. Surg. 140, 1294 1299.e1 (2010).

[18] Yanbin, W. Y. W., Deqiang, S. D. S., Peng, L. P. L. and Ya, G. Y. G., "Pectus Excavatum Nuss Orthopedic finite element simulation," Biomed. Eng. Informatics (BMEI), 2010 3rd Int. Conf. 3 (2010).

[19] Zhong, W., Ye, J., Liu, J., Zhang, C. and Zhao, M., "Numerical Simulation and Clinical Verification of the Minimally Invasive Repair of Pectus Excavatum,” Open Biomed. Eng. J. 8(1), 147-152 (2014).

[20] Nagaso, T., Miyamoto, J., Kokaji, K., Yozu, R., Jiang, H., Jin, H. and Tamaki, T., "Double-bar application decreases postoperative pain after the Nuss procedure,” J. Thorac. Cardiovasc. Surg. 140(1), 39-44.e2 (2010).

[21] Rechowicz, K. J. and McKenzie, F. D., "Development and validation methodology of the Nuss procedure surgical planner," Simulation, 0037549713496153- (2013).

[22] Ye, J.-D., Lu, G.-P., Feng, J.-J. and Zhong, W.-H., "Effect on Chest Deformation of Simultaneous Correction of Pectus Excavatum with Scoliosis.,” J. Healthc. Eng. 2017, 8318694 (2017).

[23] Xie, L., Cai, S., Xie, L., Chen, G. and Zhou, H., "Development of a computer-aided design and finite-element analysis combined method for customized Nuss bar in pectus excavatum surgery," Sci. Rep. 7(1), 3543 (2017).

[24] AWREJCEWICZ, J. . Ł. B., "DYNAMICS OF THE HUMAN THORAX WITH THE LORENZ PECTUS BAR,” 22nd Int. Conf. Vib. Phys. Syst., Poznań-Będlewo, Poland (2006).

[25] Li, Z., Kindig, M. W., Subit, D. and Kent, R. W., "Influence of mesh density, cortical thickness and material properties on human rib fracture prediction.," Med. Eng. Phys. 32, 998-1008 (2010).

[26] Guo, B., Liao, D., Li, X., Zeng, Y. and Yang, Q., “Age and gender related changes in biomechanical properties of healthy human costal cartilage.," Clin. Biomech. (Bristol, Avon) 22, 292-297 (2007).

[27] Qing-Hua, Y., Yu-Peng, S., Haiyue, J. and Hong-Xing, Z., "The significance of the biomechanical properties of costal cartilage in the timing of ear reconstruction surgery.," J. Plast. Reconstr. Aesthet. Surg. 64, 742-746 (2011).

[28] Forman, J. L. and Kent, R. W., "Modeling costal cartilage using local material properties with consideration for gross heterogeneities.," J. Biomech. 44, 910-916 (2011).

[29] Lapeer, R. J., Gasson, P. D. and Karri, V., “A Hyperelastic Finite-Element Model of Human Skin for Interactive Real-Time Surgical Simulation,” IEEE Trans. Biomed. Eng. 58(4), 1013-1022 (2011).

[30] Kikinis, R., Pieper, S. D. and Vosburgh, K. G., "3D Slicer: A Platform for Subject-Specific Image Analysis, Visualization, and Clinical Support," [Intraoperative Imaging and Image-Guided Therapy], Springer New York, New York, NY, 277-289 (2014)

[31] Fang, Q. F. Q. and Boas, D. A., “Tetrahedral mesh generation from volumetric binary and grayscale images," 2009 IEEE Int. Symp. Biomed. Imaging From Nano to Macro (2009).

[32] Vilaça, J. L., Rodrigues, P. L., Soares, T. R., Fonseca, J. C., Pinho, A. C., Henriques-Coelho, T. and CorreiaPinto, J., "Automatic Prebent Customized Prosthesis for Pectus Excavatum Minimally Invasive Surgery Correction.," Surg. Innov. 21, 290-296 (2013).

[33] Neves, S. C., Pinho, A., Fonseca, J. C., Rodrigues, N. F., Henriques-Coelho, T., Correia-Pinto, J. and Vilaça, J. L., "Finite element analysis of pectus carinatum surgical correction via a minimally invasive approach," Comput. Methods Biomech. Biomed. Engin. 18(7), 711-720 (2015).

[34] Rack, H. and Qazi, J., “Titanium alloys for biomedical applications,” Mater. Sci. Eng. C 26, 1269-1277 (2006).

[35] Ye, J., Wang, Z., Zhang, C. and Liu, J., "The numerical modeling to the orthopedic of thorax with pectus excavatum,” 2011 Int. Conf. Consum. Electron. Commun. Networks, 5417-5420 (2011).

[36] Yu, H. Y., Cai, Z. B., Zhou, Z. R. and Zhu, M. H., "Fretting behavior of cortical bone against titanium and its alloy," Wear 259(7-12), 910-918 (2005).

[37] Moreira, A. H. J., Gomes-Fonseca, J., Rodrigues, P. L., Fonseca, J. C., Pinho, A. C. M., Correia-Pinto, J., Rodrigues, N. F. and Vilaça, J. L., "Assessment of 3D Scanners for Modeling Pectus Carinatum Corrective Bar," Proc. Int. Conf. Comput. Vis. Theory Appl., 122-125, SciTePress - Science and and Technology Publications, Barcelona (2013).

[38] Gomes-Fonseca, J., Vilaça, J. L., Henriques-Coelho, T., Direito-Santos, B., Pinho, A. C. M., Fonseca, J. C. and Correia-Pinto, J., "A new methodology for assessment of pectus excavatum correction after bar removal in Nuss procedure: Preliminary study,” J. Pediatr. Surg. 52(7), 1089-1097 (2017). 